\title{
Adaptation of activity-based-costing (ABC) to calculate unit costs in Mental Health Care in Spain
}

\author{
Karen Moreno \\ Department of Accounting and Finance, \\ Universidad Complutense de Madrid \\ SPAIN
}

\begin{abstract}
Background: To date, numerous cost-of-illness studies have been using methodologies that don't provide trustworthy results for decision making in mental health care.

Objectives: The aims of this paper are design and implement a cost methodology by process of patient's care to calculate unit costs in mental health in Spain in 2005 and compare the results with the reached ones by traditional methods.

Methods: We adapted Activity-Based-Costing to this field analyzing the organizational and management structure of Mental Health's public services in a region of Spain, Navarre, describing the processes of care to patient in each resource and calculating their cost.

Results: We implemented this methodology in all resources and obtained unit cost per service. There are great differences between our results and the ones calculated by traditional systems. We display one example of these disparities contrasting our cost with the reached one by the methodology of Diagnostic Related Group (DRG).

Conclusions: This cost methodology offers more advantages for management than traditional methods provide.
\end{abstract}

Received 27 March 2006

Accepted 14 July 2006

\section{Background}

In last years, there has been a great concern about cost-of-illness studies in mental health care, since these papers are not using a solid cost methodology and so are not obtaining trustworthy results ${ }^{1-11}$ and besides, the results obtained until now and the design of management tools based on them are quite little ${ }^{10}$

There are many deficiencies of traditional cost methodologies utilized to date; some studies calculate the total expenditure of patient's care for a certain mental pathology, but they don't obtain unit costs per patient or per service; this fact doesn't allow using the results for cost-effectiveness analysis or in 
economic evaluations or in decision making about financing for example ${ }^{12-16}$. However, there are papers that calculate unit costs per patient or service but, most of them, are using tariffs or prices previously established; these figures are taken as valid but with their use, average costs are obtained that do not reveal the totality of emaciated resources by type of patient ${ }^{12,17-23}$. In other cases, if unit costs are calculated, the budgetary expenditure has been taken as the total consumption of resources in each service, and unit costs are the result of dividing that amount between the number of patients who have used the service; this average cost is not correct since the consumption of resources will be different according to patient's pathology; let's think about one Mental Health Centre, a schizophrenic patient will receive treatment mainly with psychiatrist and nurse, but a depressive person will spend more time with psychologist, so the cost of both people will be completely different. Moreover, there are many concepts, like amortizations of an investment, that are not included in the budgetary expenditure and so that cost calculated according to it, is not complete.

Due to the previously stated, it's necessary to design a solid methodology; it must be easy to implant, it should make possible the comparison at international level and provide reliable results for being used in any kind of economic evaluation (cost-effectiveness analysis, efficacy analysis, decision making about financing...); this is exactly our main aim of our paper, to get this methodology and compare our results with the reached ones by traditional methods.

\section{Methods}

We are going to analyze all schizophrenic patients (1,300 people) living in Navarre, region in the North of Spain with 600,000 habitants, because of relevance of this pathology $y^{2,4,5,7,13}$. We will evaluate all Mental Health's public services that specifically are: 9 Mental Health Centres, 2 Psychiatric Wards in Hospital, 2 Day-Hospitals, a Rehabilitation Clinic with two types of services provided (part-time hospitalization and day-centre) and a Long and Medium Term Residential Care.

Some instruments will be utilized in the development of this research: one of them is the European Service Mapping Schedule (ESMS), system of standardization of resources presented in 2000 and later adapted in some zones of Spain and specifically in Navarre ${ }^{24-26}$. Furthermore, we will compare our methodology with the traditional cost system used in each service: for example, we will use DRG system, tariffs fixed by Navarre's Government for some services or simply unit costs calculated by the staff of the resources.

We proceeded of this way: first of all, we analyzed the organizational and management structure of Mental Health's public services. With the consecution of this stage we had a clear vision of available services for patients and we knew how these services were related; this phase was carried out with a focal point with Director of Mental Health's area in Navarre, the person who better knows the framework of mental health in the region, who gave details of all Mental Health's public services during three meetings of 45 minutes approximately. Having all these data, we resorted of ESMS codes and we checked that all services were codified with the right code.

After, having an exhaustive knowledge about services, we followed having focal points, but in this case with personnel of each kind of service; maintaining 2 interviews of 30 minutes approximately with them, we managed to design the processes that identify care and management of each resource. These processes are identified with the group 
of activities that cause expenses and represent a care's protocol of the service; in this way, we have developed a care's model for patients through the design of processes and the list of activities that compose them. For validating these models for each service, we formed a focal group, one per service with the directors, and 2 meetings were of one hour long. Finally we reached a consensus about these care's models.

Once we have this map of activities designed for each service, the next step consisted of allocation of resources to each process for being able to calculate unit costs. This task was not complicated because we had at our disposal all necessary information; we calculated unit costs per process and after, with this data, we easily obtained cost per patient by means of the aggregation of cost of processes that each patient had needed.

With all this data, we proceeded to design a spreadsheet for each service that can be updated next years without any complication.

\section{Results}

This methodology was implemented in all Mental Health's public services in Navarre; we have obtained very good results and unit costs per patient were calculated for all services in 2005 and were compared with the ones reached by traditional methodologies obtaining important differences. As one example of these outcomes, we display results for a specific service; we have chosen the Psychiatric Ward in Hospital because we can show differences in costs between our results and D.R.G. methodology that it's commonly accepted and used 17,27,28. This resource is codified with R2, code of European Service Mapping Schedule (ESMS); in Figure 1, we show the organizational and management structure of Mental Health's public services. As we can observe, this service is taking care of patients coming from Mental Health Centres (80\%), Day-Hospitals (5\%) or Emergency Department (15\%) and people discharged from it will go to Mental Health Centres, DayHospitals or Rehabilitation Clinic.

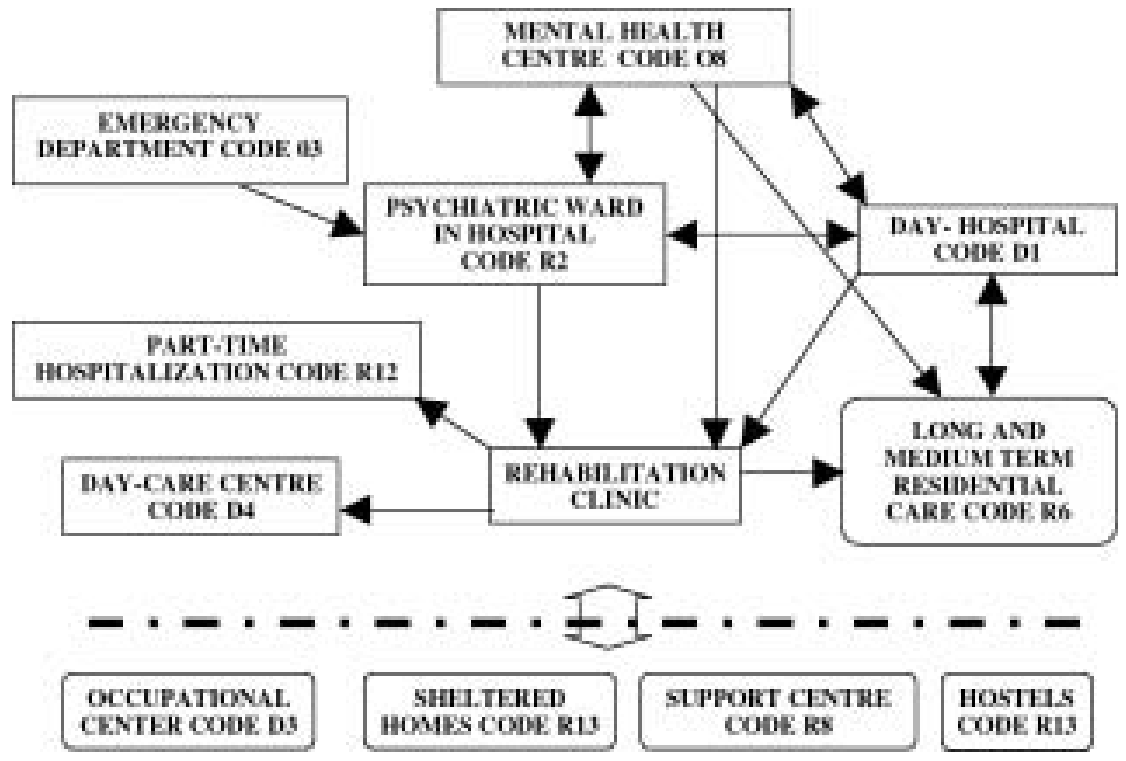

Figure 1. Map of Mental Health's Public Services in Navarre with links between them and ESMS codes. 
The design of care's processes to patient in this service can be watched on Figure 2 according to the protocol of care to patient just as the focal group set. The description of the activities that compose each process and the allocation of resources implied in each one are displayed on Table I.

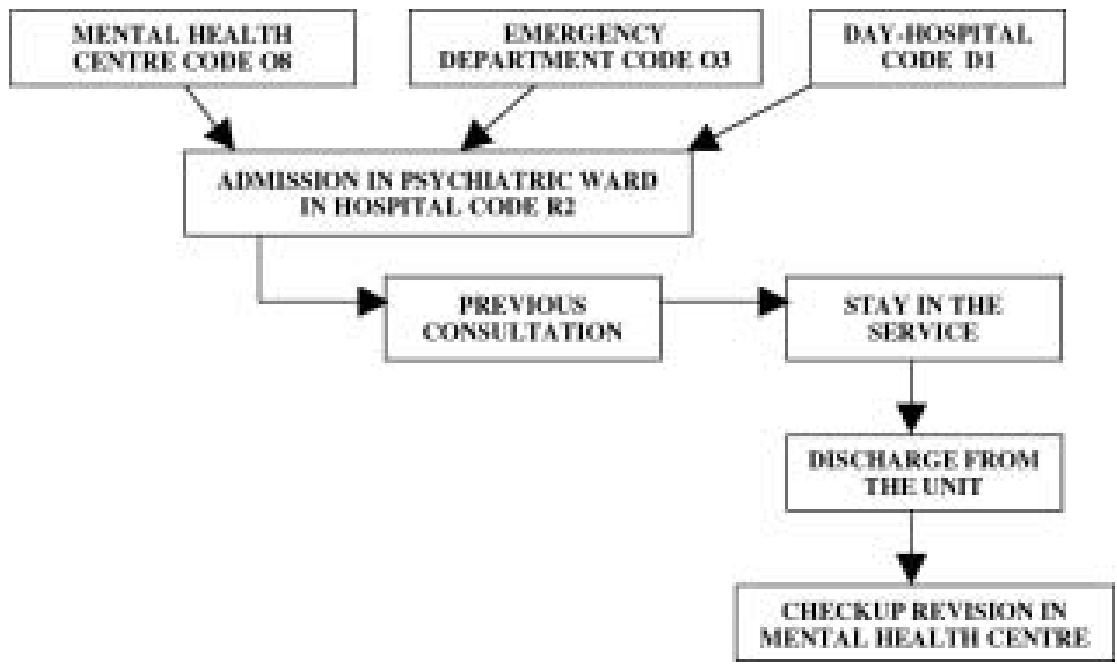

Figure 2. Design of care's processes to patient in Psychiatric Ward in Hospital in Navarre for schizophrenic patients.

Table I

Description of care's processes to schizophrenic patients in Psychiatric Ward in Hospital and allocation of necessary resources to carry out them.

Processes Description

Personnel

Length

Admission in the service.

Previous consultation.

Stay in the unit.

Blood general test.

Interview with relatives.

Patient daily consultation.

Feeding.

Medicine treatment.

Daily care.

Checkup consultation.
Patient's data registry.

Evaluation consultation for diagnose and decide the treatment.

Blood general test for detecting if there is abuse substance or something like that.

Interview with relatives.

Revision daily consultation for evaluating the patient's state.

Catering given to the patients.

Specific unidose consumed by each patient.

Daily care given to patients

by morning shift.

Daily care given to patients

by afternoon shift.

Daily care given to patients

by night shift.

Checkup consultation after patient is discharged.
Administrative Official 10 minutes

Psychiatrist. 1 hour

Nurse, laboratory

personnel.

Social worker. $\quad 45$ minutes

Psychiatrist. 30 minutes

$-$

3 nurses, 6 clinic assistants. 7 hours

4 nurses, 7 clinic assistants. 7 hours

Nurse, 2 clinic assistants. 10 hours

Mental Health Centre's 30 minutes psychiatrist. 
The differences between $\mathrm{ABC}$ cost and DRG cost, group 430, is quite considerable just as Table II shows. We have considered a patient who stayed 17 days in this service in 2005 .

Table II

Unit cost per process and per schizophrenic patient in Psychiatric Ward in Hospital and cost of DRG 430.

Processes

Unit costs $(€) 2005$

\begin{tabular}{lr}
\hline Admission in service & \\
Previous consultation & 40 \\
Stay & 20 \\
$\quad$ Blood general test & 35 \\
Interview with relatives & 330 \\
Patient's consultation & 95 \\
Feeding & 180 \\
Medicine treatment & 2,100 \\
Daily care & 20 \\
Revision consultation & 205 \\
Common costs (laundry, sterilisation...) & $3,025 €$ \\
Total cost & $5,105 €$ \\
DRG 430 Cost &
\end{tabular}

\section{Discussion}

Faced with the necessity of an improved methodology to calculate unit costs in mental health care, we have implemented ABC system obtaining results very different from the ones reached by traditional methods; in this way we have obtained cost per care's process since this alternative was already suggested as the best option but it had never been carried out ${ }^{27,28}$.

ABC system presents many advantages against traditional ones. With our methodology we have got a better knowledge about cost components just as Table II shows. As processes' cost is detailed, we can know which of them supposes a bigger consumption of resources; however, with traditional methodologies we are not able to have at our disposal this information.

A second advantage of $\mathrm{ABC}$ methodology is that unit cost contains all necessary resour- ces; let's remember that traditional methodologies are based on budgetary expenditure that is not complete because some concepts like amortizations are not included.

Moreover, this methodology makes possible cost calculation according to different temporal horizons; that is, we can calculate unit cost in a specific service per day, per month, per year or per stay; for example, in Table II we have calculated the cost for a stay of 17 days long, but if our patient had stayed 25 days the cost would be different and its calculation is very easy; this possibility is impossible with traditional systems as DRG in this case only gives one figure for all patients included in 430 group. This problem with DRG methodology has been already stated for many health's areas and Psychiatry is not an exception ${ }^{27}$.

As well, it's worth mentioning that $\mathrm{ABC}$ methodology is not only an instrument to get unit costs of care based in process. This 
method links cost calculation with the activities that characterize each process; for this reason, this system becomes a management tool very familiar and of easy understanding for service's personnel and directors since the list of activities of each process is an accurate reflection of work in each service. In this way, we can contrast the protocol of care's processes between areas from a medical point of view and perhaps improve care's patient.

In addition, we can affirm that if our cost is more analytic and reliable, many type of researches (cost-effectiveness analysis, efficacy analysis, comparisons between areas or any kind of economic evaluation) will improve their results; their conclusions will be more coherent than works made according to traditional costs.

Finally, one of the most important advantages of $\mathrm{ABC}$ methodology is based on the improvement of decision making about services' financing, provision of new services, reorganization of services... If we have at our disposal a better knowledge about cost components, unit cost per process, patient (differentiating for pathologies) and service as a whole, we are able to make a better decision than if we only know the traditional cost.

In spite of these properties, this methodology shows an important limitation that lies on the data compilation. If services' personnel and directors don't collaborate and don't help us to design and describe care's processes and allocate resources on them, this methodology can not be implemented. Without this closed collaboration, key element as it has been stated in previous papers $^{29}$, this system does not work. But as we have demonstrated, multidisciplinary work increases possibilities of success in the obtaining of trustworthy results.

Another limitation of this study is that we have only implemented $\mathrm{ABC}$ methodology in a region; it should be very interesting if we carried out the same work in another area for validating the use of ABC methodology in mental health care.

\section{Acknowledgements}

This paper belongs to a research project funded by Fondo de Investigación Sanitaria (FIS), PI040077, and also to RIRAG, research network funded by the same Funding Body.

\section{References}

1. Becker T, Kilian R. Psychiatric services for people with severe mental illness across Western Europe: what can be generalized from current knowledge about differences in provision, costs and outcomes of mental health care? Acta Psychiat Scand 2006; 113(Suppl.429): 9-16.

2. Grover S, Avasthi A, Chakrabarti S et al. Cost of care of schizophrenia: a study of Indian out-patient attendees. Acta Psychiat Scand 2005; 112(1): 54-63.

3. Urdahl H, Knapp M, Edgell ET et al. Unit costs in international economic evaluations: resource costing of the Schizophrenia Outpatient Health Outcomes Study. Acta Psychiat Scand 2003; 107(Suppl.416): 41-47.

4. Knapp M, Chisholm D, Leese M et al. Comparing patterns and costs of schizophrenia care in five European countries: the EPSILON study. Acta Psychiat Scand 2002; 105(1): 42-54.

5. Salize HJ. Costs of schizophrenia-what we know (not)? Psychiat Prax 2001; 28(Suppl.1): S21-S28.

6. Saldivia S. Estimación de costes de la esquizofrenia asociado al uso de servicios. Thesis. Granada: 2000.

7. Agustench C, Cabasés JM and PSICOST Group. Análisis y costes de utilización de servicios de la esquizofrenia en Navarra durante los tres primeros años de la enfermedad. An Sist Sanit Navarra 2001; 23 (Suppl. 1): S83-S94.

8. Becker T, Knapp M, Knudsen HC et al. The EPSILON study of schizophrenia in five European countries. Design and methodology for standardising outcome measures and 
comparing patterns of care and service costs. Brit J Psychiat $1999 ; 175: 514-521$.

9. McCrone P, Thornicroft G, Phelan M et al. Utilisation and costs of community mental health services. PRiSM Psychosis Study. Brit J Psychiat 1998; 173: 391-398.

10. McCrone P, Weich S. Mental health care costs: paucity of measurement. Soc Psych Psych Epid 1996; 31(2): 70-77.

11. Davies LM, Drummond MF. Economics and schizophrenia: the real cost. Brit J Psychiat 1994; Suppl. 25: S18-S21.

12. Carr VJ, Neil AL, Halpin SA et al. Costs of schizophrenia and other psychoses in urban Australia: findings from the Low Prevalence (Psychotic) Disorders Study. Aust NZ J Psychiat 2003; 37(1): 31-40.

13. Knapp M, Mangalore R, Simon J. The global costs of schizophrenia. Schizophrenia Bull 2003; 30(2): 279-293.

14. Fattore G, Percudani M, Pugnoli C et al. Organisational structure, routine clinical activity and costs of a community psychiatric service in Lombardy region. Int $\mathrm{J}$ Soc Psychiatr 2000; 46(4): 250-264.

15. Shah A, Jenkins R. Mental health economic studies form developing countries reviewed in the context of those from developed countries. Acta Psychiat Scand 2000; 101: 87-103.

16. Rund BR, Ruud T. Costs of services for schizophrenic patients in Norway. Acta Psychiat Scand 1999; 99(2): 120-125.

17. Garattini L, Barbui C, Clemente R et al. Direct Costs of Schizophrenia and Related Disorders in Italian Community Mental Health Services: A Multicenter, Prospective 1 Year Followup Study. Schizophrenia Bull 2004; 30(2): 295-302.

18. Rogers D, Covelli G, Garattini L. I costi diretti dei servizi psichiatrici in un dipartimento di salute mentale. Farmeconomia e Percorsi Terapeutici 2001; 2: 5-9.

19. Tarricone R, Gerzeli S, Montanelli R et al. Direct and indirect costs of schizophrenia in community psychiatric services in Italy. The GISIES study. Health Policy 2000; 51: 1-18.

20. McCrone P, Menezes PR, Johnson S et al. Service use and costs of people with dual diagnosis in South London. Acta Psychiat Scand 2000; 101: 464-472.

21. Salvador-Carulla L, Haro JM, Cabasés J et al. Service utilization and costs of first-onset schizophrenia in two widely differing health service areas in North-East of
Spain. PSICOST Group. Acta Psychiat Scand 1999; 100(5): 335-343.

22. Salize HJ, Rossler W. The cost of comprehensive care of people with schizophrenia living in the community. A cost evaluation from a German catchment area. Brit $\mathrm{J}$ Psychiat 1996; 169(1): 42-48.

23. Dickey B, Beecham J, Latimer E, Stephen H. COMMUNITY SUPPORT: the Evaluation Center@HSRI Toolkit. Estimating Per Unit Treatment Costs for Mental Health and Substance Abuse Programs. USA: 1999. http://www.mentalhealth.samhsa.gov/cmhs/CommunitySupplport/research/toolkits/pn37ch1.asp

24. Johnson S, Kuhlmann R, EPCAT Group. The European Service Mapping Schedule (ESMS): development of an instrument for the description and classification of mental health services. Acta Psychiat Scand 2000; 102(Suppl.405): 14-24.

25. Salvador-Carulla L, Romero C, Martínez A et al. Assessment instruments: standardization of the European Service Mapping Schedule (ESMS) in Spain. Acta Psychiat Scand 2000; 102(Suppl.405): 24-33.

26. Beperet M, Nadal S, Martínez A et al. Diagrama Europeo de Servicios de Salud Mental y su aplicación en el sector IB de Burlada. An Sist Sanit Navarra 2000; 23(Supl.1): S53-S62.

27. Seva-Díaz A, Seva-Fernández A. Los GRDs psiquiátricos: Una investigación pendiente. Eur J Psychiat 2003; 17(1): 49-63.

28. Seva A. El coste de las patologías psiquiátricas en España: un seguimiento de 26 años y 10.794 ingresos en una Unidad Psiquiátrica de Corta Estancia de un hospital general. Eur J Psychiat 2002; 16(1): 57-67.

29. Moscarelli M. Health and economic evaluation in schizophrenia: implications for heath policies. Acta Psychiat Scand 1994; 89(Suppl.382): 84-88.

Address for correspondence:

Karen Moreno

Universidad Complutense de Madrid

Campus de Somosaguas

Ed. Prefabricado, $3^{\circ}$ planta

28223 Pozuelo de Alarcón

Madrid, Spain

Ph: +34-91-3942357

Fax: +34-91-3942381

e-mail:kmorenoo@ccee.ucm.es 